\title{
EVOLUCIÓN, AVANCES Y PERSPECTIVAS DE LA INVESTIGACIÓN EN UNA UNIVERSIDAD PEDAGÓGICA VENEZOLANA
}

\author{
José Alberto Peña ${ }^{1}$ \\ Instituto Pedagógico de Miranda José Manuel Siso Martínez - Venezuela \\ Grupo de investigación HISULA \\ joseal@cantv.net \\ Miren De Tejada Lagonell ${ }^{2}$ \\ Instituto Pedagógico de Caracas- Venezuela \\ Grupo de investigación HISULA \\ tejadalagonell@gmail.com
}

Recepción: 05/05/2011

Evaluación: 28/06/2011

Aceptación: 22/11/2011

Artículo de Reflexión

\section{RESUMEN}

En el presente artículo se aborda la temática correspondiente a la evolución, avances y perspectivas de la Investigación en la Universidad Pedagógica Experimental Libertador (UPEL) de Venezuela. Se plantea como una indagación de carácter documental y descriptiva, cuyo propósito es exponer el estado actual de la investigación en la UPEL. Con tal fin, se revisan aspectos históricos, estructurales y de pertinencia de la investigación. Se encuentra que en el devenir histórico la investigación en dicha universidad, se aprecian elementos que han aletargado esta función, palpable en la caracterización de los trabajos de investigación; sin embargo, la presencia de instrumentos legales y la constante reformulación de las Políticas de financiamiento de la investigación le han dado un nuevo sentido e impulso. De igual forma

1 Doctor en Ciencias de la Educación. Profesor Titular de la Universidad Pedagógica Experimental Libertador en el Instituto Pedagógico José Manuel Siso Martínez. Investigador Consolidado adscrito al Núcleo de Investigación en Creatividad "Dr. David Vivas".

2 Doctora en Psicología. Profesor Asociado de la Universidad Pedagógica Experimental Libertador en el Instituto Pedagógico de Caracas. Investigadora Consolidada adscrita al Instituto de Investigaciones Lingüísticas y Literarias Andrés Bello. 
se establece que una de las variables favorecedoras para dicha actividad, es la estructura organizativa y gerencial que administra la investigación en dicha Universidad (Vicerrectorado de Investigación y Postgrado a nivel del Rectorado y las Subdirecciones de Investigación y Postgrado a nivel de los Institutos). Para dar respuesta a la pertinencia, caracterizamos en cinco categorías las investigaciones realizadas en la UPEL, las cuales son expuestas de manera sucinta. Por último, se muestran algunos indicadores que señalan que la investigación en esta Universidad tenía una tendencia a fortalecerse, antes del recorte presupuestario del año 2008. Se presentan lineamientos generales para superar ese letargo y avanzar hacia su consolidación.

Palabras clave: Revista Historia de la Educación Latinoamericana, Universidad Pedagógica, pertinencia social, unidades de investigación, investigadores.

\title{
EVOLUTION, ADVANCES AND PERSPECTIVE OF THE INVESTIGATION IN A VENEZUELAN PEDAGOGICAL UNIVERSITY
}

\author{
José Alberto Peña \\ Instituto Pedagógico de Miranda José Manuel Siso Martínez- Venezuela \\ Research group HISULA \\ joseal@cantv.net \\ Miren De Tejada Lagonell \\ Instituto Pedagógico de Caracas- Venezuela \\ Research group HISULA \\ tejadalagonell@gmail.com
}

\begin{abstract}
The present article approaches about thematic that bellows to the evolution, advances and perspective of the Investigation in the Pedagogical University Experimental Liberator (UPEL). It considers like investigation of documentary and descriptive character, whose intention is to exhibit the present state research in the UPEL. With such aim historical aspects are reviewed, structural and of relevance of the research. One is that in historical happening of the investigation in the university, elements are appraised that have made drowsy this function, concrete in the characterization of the works of research; nevertheless, the presence of legal instruments and the constant reformulation of the policies of financing of the research has
\end{abstract}


given to a new sense and impulse it. Similarly one settles down that one of the enhancing variables of the research, is the organizational structure and managemental of the research in the university. In order to give an answer to relevance, we characterized in five categories the research realized in the UPEL, which is exposed in a brief way. Finally, there are some aspects that indicate that the research in our University tends to fortify itself, and ideas for their advance and consolidation.

Key words: Journal of Latin American Education History, pedagogical University, social relevance, units of investigation, investigators.

\title{
EVOLUÇÃO, AVANÇOS E PERSPECTIVAS DA PESQUISA EM UMA UNIVERSIDADE PEDAGÓGICAVENEZUELANA
}

\author{
José Alberto Peña \\ Instituto Pedagógico de Miranda José Manuel Siso Martínez- Venezuela \\ Grupo de pesquisa HISULA \\ joseal@cantv.net \\ Miren De Tejada Lagonell \\ Instituto Pedagógico de Caracas-Venezuela \\ Grupo de pesquisa HISULA \\ tejadalagonell@gmail.com
}

\section{RESUMO}

No presente artigo se aborda a temática correspondente à evolução, avanços e perspectivas da Pesquisa na Universidade Pedagógica Experimental Libertador (UPEL) de Venezuela. Apresenta-se uma indagação de caráter documental e descritiva, cujo propósito é expor o estado atual da pesquisa na UPEL. Com tal finalidade, são revisados aspectos históricos, estruturais e de pertinência da investigação. Percebe-se que no devenir histórico a pesquisa na dita universidade, apreciam-se elementos que letargiaram esta função, palpável na caracterização dos trabalhos de pesquisa; todavia, a presença de instrumentos legais e a constante reformulação das Políticas de financiamento da pesquisa lhe deram um novo sentido e impulso. De igual forma, se estabelece que uma das variáveis favorecedoras para a dita atividade é a estrutura organizativa e gerencial que administra a pesquisa na dita Universidade (Vice-reitorado de Pesquisa e Pos-graduaçãoestrutura organizativa e gerencial que administra a pesquisa na dita Universidade (Vice-reitorado de Pesquisa e Pos-graduaçlsi 
Palavras-chave: Revista História da Educação Latino-americana, Pedagogical University, social relevance, units of investigation, investigators.

\section{INTRODUCCIÓN}

Uno de los objetivos de la Educación Superior ${ }^{3}$ en Venezuela, es fomentar la investigación de nuevos conocimientos e impulsar el progreso de la ciencia, la tecnología, las letras, las artes y demás manifestaciones creadoras del espíritu, en beneficio del bienestar social. Por su parte, la Ley de Universidades de Venezuela ${ }^{4}$, señala que para cumplir su función rectora en la educación, la cultura y la ciencia, las universidades orientarán sus actividades a crear, asimilar y difundir el saber mediante la investigación y la enseñanza, las cuales son funciones obligantes para las universidades nacionales; de manera particular, la Universidad Pedagógica Experimental Libertador (UPEL) a través de las actividades investigativas desarrolladas por sus profesores, ha generado un cuerpo de conocimientos pertinentes que dan respuestas a las necesidades que tiene el país en el campo de las Ciencias Sociales y las Humanidades y, más específicamente, en el de la Educación; dichas funciones se desarrollan de conformidad con el conjunto de lineamientos derivados de las Políticas de Investigación formuladas por la Universidad ${ }^{5}$.

De esta manera los miembros del cuerpo profesoral de la Universidad Pedagógica Experimental Libertador, basados en valores como: calidad, respeto, responsabilidad, equidad, solidaridad, honestidad, se comprometen en cumplir con una función fundamental del ser docente: hacer investigación. En este artículo, considerando como centro de interés la función investigación en la UPEL, se pretende revisar el devenir histórico de la misma, apreciar la situación actual y hacer proyecciones sobre su perspectiva de cara a un recorte presupuestario (por Políticas del Gobierno) que la amenazan seriamente.

3 Señalado explícitamente en el artículo 27, literal 2 de la Ley Orgánica de Educación en Venezuela (1980).

${ }^{4}$ LEY DE UNIVERSIDADES. (1970): Disponible: http://www.ucab.edu.ve/ tl_files/Reglamentos/1.01.pdf [Consulta: 2011, Enero 05].

5 La UPEL (2007): entre otras, tiene como Misión “...la formación, capacitación, perfeccionamiento y actualización de docentes de elevada calidad personal y profesional, y...la producción y difusión de conocimientos socialmente válidos, que desarrolla procesos educativos con un personal competente, participativo y comprometido con los valores que promueve la universidad. Asimismo, la UPEL aspira a convertirse en "....una universidad de excelencia, comprometida con el país para elevar el nivel de la educación, mediante la formación de docentes competentes; capaces de generar conocimientos útiles para implementar procesos pedagógicos innovadores e impulsar la transformación de la realidad social, y así contribuir al logro de una sociedad más próspera, equitativa y solidaria". 
En síntesis, la intención es brindar a la comunidad académica universitaria nacional y latinoamericana, una visión de la configuración y desempeño de la función investigación en una universidad pública venezolana de formación docente.

\section{Algunos criterios para el análisis de la función investigación en la Universidad Pedagógica Experimental Libertador}

Existen interrogantes que, recurrentemente, los docentes se plantean al reunirse en eventos de investigación institucional, local, regional o nacional, cuando surge como tema de conversación la "investigación en la UPEL"; algunas de tales preguntas suelen ser, entre otras las siguientes $\_$En el entorno académico universitario, somos reconocidos como investigadores?; ¿Cuál es el impacto social de las investigaciones que se desarrollan en la UPEL?; $¿$ ¿Nos reconocemos como poseedores de una cultura investigativa?

Preguntas de este tipo generan reflexiones que conducen a analizar y/o comprender la función investigación que se desarrolla en la UPEL.

En la búsqueda de las respuestas, es preciso considerar aspectos de carácter histórico para examinar el proceso que ha seguido la actividad investigativa en la Universidad; componentes estructurales, que permitan dilucidar cómo se configura y ordena la investigación en la UPEL y elementos vinculados a la pertinencia de la investigación, que ayuden a develar el alcance e impacto de ésta ${ }^{6}$. Aún cuando haremos énfasis en estos tres criterios, no dejamos de reconocer la existencia de otros que, relacionados con el ámbito investigativo dentro de nuestra Universidad, ayudan a responder las interrogantes planteadas; entre ellos los referidos a la calidad de las investigaciones o a la formación de los investigadores.

\section{Aspectos históricos relacionados con la función investigación en la UPEL}

Govea de Carpio $^{7}$, Barrios ${ }^{8}$ y Peñalver ${ }^{9}$, precisan cómo ha sido la formación docente en Venezuela, aspecto éste que toca en todas sus

6 PEÑA, José. (2008): "Perspectivas de la Investigación en la UPEL” Ponencia presentada en el foro "Gestión de la Calidad en Educación"; XII Jornada de Investigación Institucional de la UPEL-IPB. Edo. Lara.

7 GOVEA DE CARPIO, Duilia. (1989): "Educación popular y formación docente, de la independencia al 23 de enero de 1958”. Caracas, Academia Nacional de la Historia, p. 23.

8 BARRIOS, Maritza. (2006): "Vocaciones y Formación de Educadores", en: Doce propuestas educativas para Venezuela, Caracas, Fundación Polar/UCAB: Fondo de Publicaciones UCAB, pp. 27-74.

9 PEÑALVER, Luis. (2007): La Formación Docente en Venezuela. Caracas, Universidad Pedagógica Experimental Libertador, Vicerrectorado de Investigación y Postgrado. 
dimensiones a nuestra Universidad, ya que está concebida como "la universidad de los maestros" y constituye la primera institución formadora de investigadores en el área de las Ciencias Sociales y de las Humanidades, con énfasis en el ámbito educativo, debido a que los docentes que en ella se forman han de estar capacitados para desarrollar procesos de observación y de investigación sistemáticos en el aula de clases, concebida como un laboratorio dinámico y humano.

La UPEL tiene como primigenio al histórico Instituto Pedagógico Nacional ${ }^{10}$-hoy Instituto Pedagógico de Caracas-, creado en 1936, por decreto del Presidente de la República General Eleázar López Contreras. Desde este momento puede ser ubicado el inicio del devenir histórico de la investigación en la UPEL ${ }^{11}$, una universidad relativamente joven si se le compara con algunas universidades nacionales fundadas en los siglos XVIII y XIX tales como la Universidad Central de Venezuela (1721), Universidad de Los Andes (1810), Universidad del Zulia (1881) y la Universidad de Carabobo (1892). Estas instituciones han contado con casi dos siglos para la construcción del prestigio que han alcanzado con base a sus avances en materia de ciencia y tecnología, consolidando una cultura investigativa y tener personal únicamente dedicado a investigar en una multiplicidad de áreas del conocimiento.

La UPEL nace sin un accionar inmediato y sólo lo hace cinco años después, el 27 de junio de 1988, cuando se firma su acta de consolidación, a la que se integran los siguientes institutos (presentados según el orden cronológico de creación): Instituto Pedagógico de Caracas (1936), Instituto de Mejoramiento Profesional del Magisterio (1950), Instituto Pedagógico de Barquisimeto (1959), Instituto Pedagógico de Maracay (1971), Instituto Pedagógico de Maturín (1971), y el Instituto Pedagógico de Miranda (1976), (Govea, 1989).

No obstante, a pesar de las buenas intenciones plasmadas en su decreto y acta fundacionales, la UPEL nace con un Reglamento de Personal

${ }^{10}$ GOVEA DE CARPIO, Duilia. (1989): Educación popular y formación docente, de la independencia al 23 de enero de 1958. Caracas: Academia Nacional de la Historia. Este instituto estaría destinado a formar los docentes para la enseñanza secundaria y normalista, así como a cooperar con el perfeccionamiento del profesorado en ejercicio, y a fomentar el estudio científico de los problemas educacionales y de la orientación vocacional, además de realizar investigaciones pedagógicas sobre educación, especialmente sobre educación venezolana.

11 PENALVER, Luis. (2007): La Formación Docente en Venezuela. Caracas, Universidad Pedagógica Experimental Libertador, Vicerrectorado de Investigación y Postgrado, p. 47. La Universidad Pedagógica Experimental Libertador, es creada el 28 de julio de 1983, mediante Decreto Presidencial No 2176 fue concebida como una "...institución para la excelencia académica, cuyo objeto sería la formación de profesionales de la docencia y especialistas en educación, la investigación y la difusión de los saberes, vinculados con las problemáticas y necesidades del sector educativo y la sociedad nacional". 
Académico (UPEL, 1997) que no fortalecío las función investigación; por ejemplo: a) para ser jurado de trabajo de ascenso lo fundamental es tener como mínimo la categoría académica a la que aspira el profesor a ascender mas no ser un investigador consolidado; otro tanto ocurre cuando se asignan tutores a los profesores instructores en período de prueba o a los estudiantes de postgrado.

Otro elemento referido a la debilidad investigativa inicial de la UPEL, es que previo a la fecha de su creación, en los Institutos los profesores ascendían por vías administrativas, de allí que por ejemplo, al analizar la base de datos del personal académico y cotejarla con la información contenida en los centros de documentación, encontramos para el año 2006 de un total de 2059 profesores jubilados en la categoría de Titular, se estima que sólo 782 (38\%) realizó algún trabajo de investigación para ascender, 187 (0.09\%) se jubilaron como profesores asistentes y 6 nunca ascendieron, es decir, se jubilaron con la categoría en la cual ingresaron a la universidad: Instructor.

En contraposición con este comportamiento inicial, en la actualidad los profesores Titulares han avanzado en el escalafón académico por trabajos de ascensos basados en el producto generado de sus investigaciones.

A modo de conclusión del primer aspecto considerado (histórico) para el análisis de la situación de la UPEL en materia investigativa, se destaca lo siguiente: primero la función investigación, en la UPEL, es relativamente nueva; cuenta con una data de 21 años, en comparación con otras universidades nacionales, segundo la labor investigativa de los docentes está en un proceso de transformación desde una actividad incipiente, hacia otra en vías de fortalecimiento.

\section{Componentes estructurales de la investigación en la UPEL Relacionados con la Institución}

El segundo aspecto, que hemos denominado estructural, está orientado a la configuración de la investigación en nuestra universidad.

Tal como se ha señalado, la investigación ${ }^{12}$ es una de las funciones inherentes al docente de la UPEL como misión y tarea fundamental. Se concibe como una actividad integrada y en interacción permanente con las

12 UPEL. (2003): El artículo 105 del Reglamento General de la UPEL, indica que: "la investigación es función esencial de la Universidad, tanto en lo referente al avance de la ciencia y de la teoría científica como en lo relativo a la aplicación del saber a la solución de problemas específicos" 
actividades de docencia y de extensión, motivada por fines tanto académicos como de servicio social.

Además, como aspecto esencial, tiene un ámbito financiero bien definido dentro de su Política presupuestaria ${ }^{13}$; aunado a ello, es importante destacar que mientras en las Universidades Autónomas y algunas Experimentales, existe un Consejo de Desarrollo Científico, Humanístico y Tecnológico (CDCHT) como un ente administrativo y rector de la investigación, la UPEL amplió este radio de acción a la academia a través de su "equivalente": el Vicerrectorado de Investigación y Postgrado (VIP), desde donde se promueven acciones orientadas al fortalecimiento de la función investigación vinculadas con el quehacer docente; es decir las investigaciones están orientadas fundamentalmente, al ámbito educacional.

En síntesis, la existencia del VIP como dependencia administrativa central, facilita el camino para articular las actividades de investigación con la docencia que se desarrolla en los Programas de Postgrados de la Universidad, y apoya el trabajo que se realiza en las Unidades de Investigación (núcleos, centros e institutos) dentro de los Institutos Pedagógicos.

La ordenación de las Unidades de Investigación en la UPEL, se rige por el Reglamento de Investigación ${ }^{14}$, formulado en atención a lo sugerido por el Consejo Nacional de Universidades de Venezuela, y recientemente modificado sobre la base de las demandas de los profesores investigadores de nuestra universidad.

Para el momento en que se escribe este artículo, la Universidad Pedagógica Experimental Libertador cuenta con una estructura investigativa conformada por un (1) Instituto de Investigación, veinte y nueve Centros (29) y sesenta Núcleos (60), en la que se encuentra inscrita 294 líneas de investigación con objetivos bien definidos y proyectos de investigación en

13 UPEL. (2009): En las políticas correspondientes al año 2008, se señalaba que a través del eje estratégico investigación, se aspira consolidar una cultura investigativa de desarrollo y creación intelectual, que impulse la generación y construcción de conocimientos para superar el carácter meramente docente, reproductor y difusor del saber al articular la docencia y la investigación, con el uso de las nuevas tecnologías de la comunicación e información (UPEL 2008); y en las Políticas presupuestarias 2009 se estima que con el proyecto Generación, Divulgación y Aplicación del Conocimiento se favorezca la investigación como soporte y fundamento del quehacer Universitario; generando nuevos conocimientos e innovaciones para propiciar los cambios y transformaciones requeridas para el mejoramiento de la calidad en la educación de la comunidad local, regional y nacional, como una contribución al desarrollo humanístico, científico, tecnológico, social y cultural del país .

14 Universidad Pedagógica Experimental Libertador. (2008): "Reglamento de Investigación”. (Resolución No 2008.316.28.19, julio 23). 
áreas referidas a: Lengua; Literatura y Lingüística; Historia y Geografía; Ciencias Naturales; Ciencias Básicas; Artes; Creatividad; Educación física, salud y recreación; Educación Ambiental; Educación Especial; Procesos Educativos; Competencias Gerenciales; Orientación y desarrollo humano; y Nuevas Tecnologías de la Información, entre otras (UPEL, 2004b; UPEL 2004c; UPEL 2004d; De Tejada, 2008; Vicerrectorado de Investigación y Postgrado, 2010).

Lo anterior evidencia la diversidad de áreas de conocimientos a la que se dedican nuestros docentes investigadores, a través de las cuales responden a las necesidades de la comunidad, de la región y del país.

\section{Relacionados con los investigadores de la UPEL}

En la Universidad Pedagógica Experimental Libertador, se define de manera expresa el Perfil del Investigador que la universidad requiere (UPEL 2004d). Así, se considera que son investigadores activos quienes publican periódicamente los resultados de sus investigaciones, consignan informes de avances de sus proyectos de investigación, son miembros activos de una Unidad de Investigación, participan como árbitro de publicaciones periódicas; actúan como tutor, asesor o jurado de proyectos vinculados con áreas de conocimiento de su competencia; son miembros de programas de reconocimiento al investigador, entre otros, (Muro, X.; Nava, M.; González, M; Giménez, F.; Becerra, O.; Calzadilla, O.; Arana, A.; Osorio, L. y Quintero, C., 2003). Se considera que este criterio, está centrado en la productividad del investigador.

Sin embargo, se propone otro criterio que de manera implícita también está presente en el perfil definido; el mismo no está relacionado ya con la productividad, sino con la experticia del investigador para emprender sus proyectos de investigación, el dominio o conocimiento de una temática que le confiera el carácter de experto y el grado de autonomía para desarrollar proyectos y formar recursos humanos para la investigación.

Dada la trascendencia de esta distinción, la Coordinación Nacional de Investigación del Vicerrectorado de Investigación y Postgrado, en trabajo conjunto con los Coordinadores Generales de Investigación de cada Instituto Pedagógico, sugirió organizar a dichos investigadores en cuatro grupos: investigadores en formación, noveles, consolidados y expertos, asignándole niveles a cada uno de ellos en función de su nivel de competencia investigativa.

Para cada nivel se exigen diferentes actividades, sin embargo como factor común se plantea estar adscrito a una Unidad de Investigación, y entre 
las diferenciadas por niveles se encuentra: ser ponente en eventos científicos de carácter nacional o internacional, publicar en revistas científicamente reconocidas y participar en la formación de recursos humanos. Tales acciones permitirían a los investigadores compartir experiencias con sus pares, así como fortalecerse y contribuir al crecimiento de las Unidades de Investigación de la Universidad ${ }^{15}$.

Sin embargo, a pesar de que se cuentan con suficientes instrumentos legales y una estructura organizativa favorable al quehacer investigativo, hasta el año 2009 sólo un 25\% de los profesores ordinarios activos de la universidad, estaban registrados como investigadores activos en Unidades de Investigación, y aproximadamente un $12 \%$, se declaraban como investigadores libres; ello nos hace pensar en un déficit interesante de considerar si se asume que todos los docentes deben ser investigadores, tal como lo manifiesta el Reglamento del Personal Académico de la UPEL ${ }^{16}$.

Ante este panorama, nos planteamos conjeturas y explicaciones sobre las razones de lo que está pasando; cualquier interrogante planteado debe estar relacionada con la cultura investigativa: ¿Podremos cambiar esta relación? ¿Qué se está haciendo o podrá hacer?

Estos interrogantes fundamentales para los objetivos de este artículo, se intentarán responder al final del mismo.

\section{Elementos vinculados con la pertinencia de la investigación en la UPEL}

El último elemento de análisis está referido a la pertinencia social de la investigación que se realiza en la UPEL; este concepto esta relacionado con lo oportuno, lo relevante y la utilidad de la investigación.

Se considera que la pertinencia de la investigación es la relación de correspondencia y adecuación del entorno universitario con las necesidades del entorno social para generar productos académicos eficaces, que se constituyan en respuestas acertadas que propicien la transformación social ${ }^{17}$.

15 ANDRADE, Arteaga; CAMPOS, de Rosa; DE TEJADA, SALGUERO, Fumero. (2006): Estos elementos fueron establecidos en una reunión técnica de Coordinadores de Investigación. Vicerrectorado de Investigación y Postgrado UPEL.

${ }^{16}$ DE TEJADA, Miren. (2008): Estructura organizativa de la investigación en la UPEL. Ponencia presentada en el evento Calidady Pertinencia de la Investigación y el Postgrado de la Universidad Pedagógica Experimental Libertador. Caracas. Trabajo no publicado.

${ }^{17}$ SOSA, Elizabeth, SÁNCHEZ. Luis Y DE TEJADA, Miren. (2010): Propuesta de Integración entre la Investigación y el Postgrado, desde la visión del Instituto Pedagógico de Caracas. Subdirección de Investigación y Postgrado. No publicado. 
En este sentido los autores del presente artículo proponen organizar las investigaciones realizadas en la UPEL en cinco categorías o bloques: a. Investigaciones en el postgrado; b. Investigaciones por el postgrado; c. Investigaciones obligantes; d. Investigaciones a modo propio; e. Investigaciones en las Unidades de Investigación. Revisemos de manera sucinta cada una de estas categorías.

\section{Investigaciones en el postgrado}

Esta primera categoría, está constituida por investigaciones cuyo objetivo final es la realización del Trabajo Especial de Grado, del Trabajo de Grado o la Tesis Doctoral, y se caracterizan por: 1. Ser realizadas por cursantes de algún Subprograma de Postgrado de la UPEL, 2. El problema de investigación, formulado por el estudiante sólo tiene relevancia para él, ya que generalmente no responde a alguna línea de investigación registrada y suelen pertenecer a líneas de investigación organizadas fuera de las Unidades de Investigación. 3. La actividad de tutoría del estudianteinvestigador es realizada fundamentalmente por un tutor, que algunas veces resulta ser personal contratado, y en ocasiones cumple un rol de asesor metodológico más que de investigador. 4. Cuando el tutor es personal de la UPEL, en oportunidades el tema del trabajo no le resulta relevante, ya que no está orientado a su particular línea de interés. 5. El producto de la investigación se coloca en el centro de documentación del Instituto.

Esta práctica no es la deseable ya que la participación en un Programa de Postgrado (Maestría y Doctorado) lleva implícita la formación de un investigador quien debe dedicar parte de su ejercicio profesional a la generación de conocimientos.

En relación a este tipo de investigaciones se considera que hay una tarea pendiente para su evaluación en términos de pertinencia académica y social; sin embargo una investigación reportada por Izarra y Escobar ${ }^{18}$ revela que al menos en el Instituto de Mejoramiento Profesional del Magisterio (IMPM), un alto porcentaje de las investigaciones realizadas conectan los objetivos de la Universidad con los del Subprograma de Postgrado al que corresponden ${ }^{19}$;

${ }^{18}$ IZARRA, Douglas y ESCOBAR, Faviola. (2007): Pertinencia de la investigación en los estudios de postgrado de la UPEL-IMPM Investigación y Postgrado. pp. 165-18.

${ }^{19}$ Ibídem., Sin embargo, estos autores acotan que “...aun cuando hay pertinencia social, pareciera ser de carácter teórico...los trabajos se limitan... a dar propuestas de solución para ser ejecutadas... hacen mayor referencia a la aplicación de conocimientos..." p. 182. 
Así mismo, se pueden mencionar los trabajos realizados por la Comisión responsable de la Evaluación de la función de investigación reportado por Muro et al $(2003)^{20}$ y la evaluación de la función de investigación en la UPEL-IPB presentada por Rojas y Pérez ${ }^{21}$, en las cuales también se da cuenta de esta situación.

\section{Investigaciones por el postgrado}

Esta categoría, a diferencia de la anterior, son las que realizan nuestros profesores cursantes en los Programas de Maestrías o Doctorados, las cuales en algunos casos, solo se realizan para cumplir con un requisito de ascenso en el escalafón académico: Magíster para ser profesor Agregado y Doctor para ser profesor Asociado; aun cuando este personal se forma para ser investigador, no existen indicios que señalen que lo serán; aún no se ha determinado el impacto del postgrado de la Universidad en el área de investigación.

\section{Investigaciones obligantes}

Las investigaciones obligantes son las que promueven los docentes por razones de necesidad personal, como los Trabajos de Ascenso; estos son investigadores ocasionales o temporales y en muchos casos desconocen o necesitan de estrategias para la investigación, todo como producto de la debilidad en los planes de desarrollo y formación para la investigación o por no asumir ésta última actividad como inherente a la misión de ser docente.

\section{Investigadores a modo propio}

Los investigadores a modo propio, mejor conocidos como investigadores libres, en muchos casos, son reconocidos por el colectivo por su alta productividad, cuentan con una multiplicidad de artículos escritos en revistas indexadas, participan como conferencistas y ponentes en eventos científicos, no dependen de los elementos organizacionales para realizar investigaciones; son formadores de una generación orientada hacia la actividad investigativa, generalmente líderes de grupos de investigación y expertos en temáticas no asociadas directamente con el área educativa, sino que este ámbito resulta ser una arista más del problema a considerar.

${ }^{20}$ MURO, Xiomara; NAVA, Minelia; GONZÁLEZ, María; GIMÉNEZ, Fanny; BECERRA, Osmery; DUBS, Renie; CALZADILLA, Omar; ARANA, Aracelis; OSORIO, Lesbia; QUINTERO, Carmen. (2003): "Hacia una definición de la calidad de la investigación en la Universidad Pedagógica Experimental Libertador”, en: Investigación y Postgrado", Vol. 18, N. 1, pp. 23-35.

${ }^{21}$ ROJAS, Blanca y PÉREZ, Isabel. (2004):’Evaluación de la función de investigación en la UPEL-IPB", en: Investigación y Postgrado, Vol. 19, N. 2, pp.145-178. 
Investigadores en las unidades de investigación

En esta última categoría se ubica el conjunto de profesores que desarrollan sus actividades de investigación de manera organizada dentro de las Unidades de Investigación de la universidad; se adscriben a líneas de investigación e inscriben proyectos de investigación, financiados o no, en las diferentes líneas, ante los cuales figuran como investigadores responsables o adscritos. El centro de su línea de acción fundamentalmente es el ámbito educativo. Igual que el grupo anteriormente descrito, cuentan con reconocimiento por su productividad académica e investigativa, tanto dentro del ámbito de la UPEL, como fuera de ella.

Es importante destacar que aunque la mayoría de las investigaciones desarrolladas desde las Unidades de Investigación están referidas al ámbito educativo, existe un grupo de investigaciones que se realizan en otras áreas del saber.

\section{Avance y fortalecimiento de la cultura investigativa en la UPEL}

Programa de Promoción al Investigador (PPI)

Una primera señal demostrativa del fortalecimiento de la investigación en la UPEL hasta el año 2008, e indirectamente del avance en su cultura investigativa, está dado por la presencia de la Universidad en el Programa de Promoción del Investigador $\left(\mathrm{PPI}^{22}\right)$.

El PPI constituye una referencia en el ámbito académico universitario y la mejor expresión de participación en el mismo, es el número de Profesores Investigadores acreditados y reconocidos por el Observatorio Nacional de Ciencia, Tecnología e Innovación (ONCTI) del Ministerio del Poder Popular para Ciencia, Tecnología e Industrias Intermedias de Venezuela.

Dicho Programa fue sometido entre los años 2009 al 2011, a un proceso de reestructuración por parte del Estado; para la fecha en que se escribe este artículo, está abierta la convocatoria para la participación a un nuevo Programa: Estimulo a la Investigación (PEI), el cual tiene lineamientos diferentes al anterior. Vale destacar que la trayectoria de los profesores que

${ }^{22}$ El PPI es un indicador de productividad investigativa en el país; tiene la bondad de ser un programa de evaluación externa a la UPEL. En el mismo, un comité de expertos en diferentes áreas del conocimiento, anualmente evalúa la productividad de los investigadores pertenecientes a universidades nacionales, institutos universitarios, academias nacionales y centros especializados de investigación, entre otros. A través del Programa se reconoce la actividad investigativa que se produce en dichas instituciones. 
pertenecían al PPI, no fue migrada al PEI, sino que ahora todos tendrán que solicitar nuevamente ingreso; con ello se pierde parte de la historia acumulada que traía este conjunto de profesores.

Sin embargo, en la evolución histórica del PPI, la contribución e incorporación de profesores de la Universidad Pedagógica Experimental Libertador había sido creciente; así puede indicarse que en un lapso de 10 años, esta aumentó de 6 investigadores para el año 1999 a 376 Profesores investigadores para el $2008^{23}$ (De Tejada, 2008, Vicerrectorado de Investigación y Postgrado, 2010).

Los siguientes gráficos son ilustrativos de lo antes señalado.

CUADRO N ${ }^{\circ} 1$

Ingreso de Profesores UPEL al PPI por año de convocatoria

\begin{tabular}{|cccccccccccc|}
\hline Año & 1.998 & 1.999 & 2.000 & 2.001 & 2.002 & 2.003 & 2.004 & 2.005 & 2.006 & 2.007 & 2.008 \\
\hline $\mathrm{N}^{\circ}$ Profes & 8 & 6 & 8 & 6 & 24 & 16 & 17 & 58 & 63 & 84 & 96 \\
\hline
\end{tabular}

El Cuadro $N^{o} 1$ muestra el número de profesores que, en cada convocatoria, se han incorporado al Programa de Promoción al Investigador (PPI) por la Universidad Pedagógica Experimental Libertador.

\section{CUADRO N ${ }^{\circ} 2$}

Evolución de la cantidad de Profesores PPI por año de convocatoria

\begin{tabular}{|cccccccccccc|}
\hline Año & $\mathbf{1 . 9 9 8}$ & $\mathbf{1 . 9 9 9}$ & $\mathbf{2 . 0 0 0}$ & $\mathbf{2 . 0 0 1}$ & $\mathbf{2 . 0 0 2}$ & $\mathbf{2 . 0 0 3}$ & $\mathbf{2 . 0 0 4}$ & $\mathbf{2 . 0 0 5}$ & $\mathbf{2 . 0 0 6}$ & $\mathbf{2 . 0 0 7}$ & $\mathbf{2 . 0 0 8}$ \\
\hline $\begin{array}{c}\text { Acumulado } \\
\mathrm{N}^{\circ} \text { Profes. }\end{array}$ & 8 & 14 & 22 & 28 & 52 & 68 & 85 & 156 & 215 & 293 & 376 \\
\hline
\end{tabular}

(Fuente: De Tejada, 2008)

El Cuadro $\mathrm{N}^{\mathrm{o}} 2$ refleja la evolución del número de Profesores UPELISTAS que participaron en el Programa PPI en los últimos diez (10) años; en las cifras están representadas no sólo las incorporaciones, sino también las renovaciones y ascensos.

A partir de las cifras y datos aportados por Cortázar ${ }^{24}$ durante la realización de la $2^{\circ}$ Reunión Ordinaria del Núcleo de los Consejo de

${ }^{23}$ DE TEJADA, Miren. (2008): "Vicerrectorado de Investigación y Postgrado". 2010.

${ }^{24}$ CORTÁZAR, José. (2008): "Programa de Promoción al Investigador del Observatorio Nacional de Ciencia, Tecnología e Innovación. Ponencia presentada en la $2^{\circ}$ Reunión Ordinaria del Núcleo de los Consejo de Desarrollo Científico, Humanístico y Tecnológico (CDCHT)". Universidad Nacional Experimental de los Llanos, Barinas, marzo, 27 y 28. 
Desarrollo Científico, Humanístico y Tecnológico (CDCHT), se puede afirmar que con la incorporación de los profesores correspondientes a la convocatoria 2008, el número de Profesores PPI de nuestra universidad, permite posicionar a la UPEL como la primera Universidad Experimental ${ }^{25}$ con el mayor número de profesores dentro del Programa y ocupar la sexta posición con relación a todas las universidades nacionales.

Con el ingreso progresivo, permanencia y ascenso de los docentes de nuestra universidad al Programa de Promoción del Investigador, empezamos a ser reconocidos en el ámbito universitario como investigadores.

La incorporación sostenida de nuestros profesores en las actividades que se ejecutan dentro del Programa PPI como la participación en las convocatorias anuales y miembro de su Comité de Experto, entre otras, constituye un reconocimiento para la UPEL dándole a la misma presencia como Investigadora en el campo de las Humanidades y las Ciencias Sociales en el país.

Por otro lado, es importante destacar que el reconocimiento como investigador, no procede sólo de la pertenencia o no al PPI, sino que además tal mérito lo otorgan las comunidades académicas correspondientes al ámbito de actuación de los investigadores. En ese sentido, vale destacar los premios y reconocimientos, que por su actuación, tienen profesores de nuestra universidad fuera de dicha instancia evaluadora de la productividad investigativa de las universidades.

\section{Revistas científicas}

Una segunda señal que permite apreciar cómo se ha venido consolidando la cultura investigativa de la universidad, es la evaluación de mérito a revistas científicas, realizada por el Fondo Nacional de Ciencia Tecnología e Información (FONACIT) ${ }^{26}$ durante el año 2009, en la que se ponderaron aspectos relacionados con: calidad de los artículos, endogamia, calidad editorial, contenido de la revista y visibilidad.

En el ranking de publicaciones correspondiente al área de Humanidades, encontramos que entre las veinte y ocho (28) revistas evaluadas, seis (6) de la

${ }^{25}$ Ibídem., Las Universidades que anteceden a la UPEL en el número de Profesores participantes son: Universidad del Zulia, Universidad de Los Andes, Universidad Central de Venezuela, Universidad Simón Bolívar y la Universidad de Carabobo.

${ }^{26}$ FONDO NACIONAL DE CIENCIA, TECNOLOGÍA E INNOVACIÓN. (2009): "Registro de Publicaciones Científicas y Tecnológicas Venezolanas" FONACIT Disponible: http://www.fonacit.gob.ve/verprintconvoca.asp?id=68 [Consulta: 2010, Enero 5]. 
UPEL calificaron favorablemente, lo cual permite que esta universidad ocupe un sitial de honor por el número y lugar que ocupan sus revistas en dicha evaluación. Las revistas de la universidad que calificaron satisfactoriamente en dicha evaluación se muestra en el Cuadro $\mathrm{N}^{\circ} 3$.

CUADRO No 3

\section{REVISTAS DE LA UNIVERSIDAD FAVORABLES EN LA EVALUACIÓN DE FONACIT}

\begin{tabular}{|ccc|}
\hline Pos & Revista & Puntaje \\
\hline 1 & LETRAS (IPC) & 82.24 \\
2 & PARADIGMA (IPMAR) & 81.98 \\
6 & TIEMPO Y ESPACIO & 78.03 \\
11 & INVESTIGACIÓN Y POSTGRADO (VIP) & 74.16 \\
16 & SAPIENS (IPMJMSM) & 69.31 \\
23 & REVISTA DE INVESTIGACIÓN (IPC) & 65.13 \\
\hline
\end{tabular}

Fuente: FONACIT (2009)

Los datos anteriormente referenciados constituyen un excelente indicador para realizar una auto evaluación en materia de cultura investigativa, si consideramos que existen más de 50 Universidades nacionales.

Unidades de investigación

Las unidades de investigación son las instancias institucionales en las cuales se desarrolla la investigación dentro de los pedagógicos de la Universidad. Se organizan a partir de las líneas de investigación y sus proyectos en relación con las particularidades investigativas de cada uno de los Institutos Pedagógicos que conforman la UPEL.

En los últimos cinco (5) años, las Unidades de Investigación de la UPEL han experimentado un incremento como producto de la organización de la investigación dentro de la universidad y de la integración de la investigación y del postgrado. Para el año 2005 la Universidad contaba con sesenta y un (61) Unidades de Investigación dentro del entorno investigativo UPELISTA; en el año 2010 dicha cifra se ha incrementado a noventa $(90)^{27}$.

El incremento en el número de Unidades de Investigación de la Universidad y la conversión de Núcleo a Centro de otras ya existentes,

${ }^{27}$ DE TEJADA, Miren, SMITTER, Yajahira y MAZZARELLA, Clemen. (2009): Vicerrectorado de Investigación y Postgrado. Informe de Gestión de la Coordinación Nacional del Programa de Investigación/Coordinación Nacional de FONDEIN. No Publicado. 
evidencia un proceso continuo de evaluación y reflexión acerca de la actividad investigativa que en ellas se genera. Asimismo, demuestra la necesidad cada vez más creciente de organizarse para generar conocimientos.

Es importante resaltar estos aspectos, ya que indican un fortalecimiento de la cultura investigativa dentro de la universidad; lo cual es producto, a su vez, de las Políticas generadas desde el Vicerrectorado de Investigación y Postgrado en los últimos diez (10) años; ello a pesar de la crisis financieras existente en las universidades nacionales en materia de investigación.

En conclusión de esta segunda parte, puede decirse que el avance en materia de investigación para la UPEL, queda expresada en: 1. Presencia creciente de profesores investigadores en Programas de reconocimiento de méritos a la investigación; 2. Ser la segunda Universidad venezolana con el mayor número de revistas con evaluación de mérito, en el área de Humanidades y 3. Incremento de Unidades de Investigación en los últimos cinco (5) años.

\section{Perspectiva de la investigación en la UPEL}

La función investigación en la UPEL, estuvo sostenida en el pasado por Políticas orientadas al apoyo y motivación hacia dicha actividad ${ }^{28}$; sin embargo, la actividad investigativa de la UPEL, en su situación actual (enero de 2011), se encuentra seriamente amenaza para su continuidad y progreso para el cumplimiento de sus metas debido a la no asignación de recursos fiscales correspondiente a los años 2009, 2010 y 2011. El negativo impacto de la deficiente situación presupuestaria ha afectado la función investigación en los siguientes aspectos:

Financiamiento de la investigación

Al revisar las Políticas del Vicerrectorado de Investigación y Postgrado, se observa que la comunidad de profesores UPELISTA había contado en los últimos 10 años con el apoyo indispensable para la realización de sus actividades de investigación; apreciable a través de:

a) Financiamiento de Proyectos de investigación a través del Fondo de Desarrollo para la Investigación (FONDEIN). Durante el período

${ }^{28}$ Estas Políticas estuvieron emplazadas a reforzar aspectos como: equidad, reconocimiento de la productividad investigativa, direccionamiento de los recursos financieros hacia las unidades ejecutoras, dotación y mantenimiento de la infraestructura de apoyo a la investigación; impulso, promoción y difusión del producto de las investigaciones, favorecer alianzas nacionales e internacionales, tal como ha quedado expresado en las Políticas del Vicerrectorado de Investigación y Postgrado correspondientes a los años 2005-2006-2007-2008. 
2005/2008, se financiaron trescientos noventa y seis (396) proyectos de investigación (De Tejada, Smitter y Mazzarella, 2009); para el año 2009 el número de proyectos financiados fue de treinta y cinco (35) y ninguno para el año $2010^{29}$.

b) Apoyo para la organización de eventos de carácter nacional en los diferentes entes académicos de la Universidad, tales como Departamentos, Cátedras y Subdirecciones /Coordinaciones de Investigación y/o Postgrados. Durante el quinquenio 2005-2010, el Vicerrectorado de Investigación y Postgrado organizó cinco (5) grandes eventos ${ }^{30}$, a saber: I Encuentro Tecnologías para la Innovación Educativa (2005); Congreso Nacional de Investigación (2006); Jornada de Reflexión: Calidad y Pertinencia de la Investigación y el Postgrado en la Universidad Pedagógica Experimental Libertador (2008); Congreso de Investigación en Educación 200831, Innovaciones en Educación: Retos y Acciones; II Encuentro de Integración de la Investigación y el Postgrado UPEL 2010. Un espacio para la transformación.

c) Financiamiento para la asistencias a eventos de carácter nacional e internacional, en los cuales participan nuestros profesores en calidad de ponentes, conferencistas, talleristas o foristas. Entre el período 2005/2009 las solicitudes de financiamiento para asistencia a eventos fueron incrementando de manera progresiva; comenzó así con apoyo a trece (13) profesores para asistencia a eventos de carácter nacional y a noventa y nueve (99) para reuniones científicas de carácter internacional. Finalizó con 429 financiamientos para el primer grupo y trescientos sesenta y cinco (365) para el segundo grupo.

A partir del año 2009 las solicitudes de financiamiento dejaron de ser atendidas por la situación presupuestaria de la Universidad. ${ }^{32}$ Actualmente existe un total de 32 solicitudes a la espera de respuesta por parte de las autoridades en la materia; sin embargo es necesario destacar, que dicha cifra debe ser mayor dado que algunos docentes investigadores deciden asistir a los eventos como ponentes, utilizando para ello sus recursos personales.

${ }^{29}$ VICERRECTORADO DE INVESTIGACIÓN Y POSTGRADO. (2010): La función de Investigación en la UPEL: pasado, presente y futuro. No publicado.

${ }^{30}$ En estas actividades científicas, fueron presentadas y discutidas por profesores UPELISTAS y de otras instituciones de Educación Superior, un promedio de ciento noventa y cuatro (194) ponencias, todas relacionadas con el ámbito de la Educación (De Tejada, Smitter y Mazzarella, 2009; Vicerrectorado de Investigación y Postgrado, 2010).

31 VICERRECTORADO DE INVESTIGACIÓN Y POSTGRADO (2008). Informe del Congreso de Investigación en Educación. Retos y Acciones. No Publicado.

32 DE TEJADA, et al. (2009): Op., cit. 
Apoyo a las Unidades de investigación

Otro elemento de interés para la proyección favorable de la universidad dentro del contexto académico nacional, está dado por el surgimiento de nuevas Unidades de Investigación ${ }^{33}$ y la conversión de Núcleos a Centros, y de Centro a Institutos, como es el caso del Instituto de Investigaciones Lingüísticas y Literarias "Andrés Bello" del Instituto Pedagógico de Caracas, el cual está solo a la espera de la Resolución del Consejo Nacional de Universidades para su aparición formal como Instituto de Investigación.

Por otro lado, se consolidaron como Unidades de Investigación los Centros de Investigación Educativa de los Institutos Pedagógico de Caracas y Mejoramiento Profesional del Magisterio, los cuales a través de la evaluación de su productividad y desempeño pasaron de una unidad administrativa, como fueron inicialmente concebidos, a una Unidad de Investigación, como lo que son realmente en la práctica universitaria diaria.

\section{Implementación del RAIP-UPEL}

La UPEL consciente que para alcanzar sus objetivos en materia investigativa es indispensable no sólo la claridad de metas, sino también la organización y conocimiento del trabajo que desarrollan sus investigadores, creó el Registro Automatizado de Investigación y Postgrado (RAIP-UPEL) ${ }^{34}$, esto proporciona la oportunidad de tener información a la vista acerca de la ubicación administrativa de los investigadores, Líneas y Proyectos de Investigación, áreas de conocimiento en las que se desempeñan, y la factibilidad de actuar como jurado, árbitro, consultor o experto en sus particulares áreas del saber.

Con el RAIP-UPEL la universidad responde oportunamente a las exigencias informativas que requieren los entes gubernamentales y el colectivo universitario para el seguimiento y evaluación de la actividad investigativa del personal académico de nuestra casa de estudios.

En los actuales momentos en el RAIP UPEL están registrados 1089 docentes ordinarios de la institución ${ }^{35}$; ello hace que exista una visibilidad

${ }^{33}$ Ibídem., El quinquenio comenzó con sesenta y una (61) Unidades de Investigación para la UPEL y concluyó con noventa (90) para el año 2010, ello a pesar de la situación presupuestaria.

34 Este es un sistema "on line" de reconocimiento e identificación de los docentesinvestigadores de la Universidad, a través del que se hace el registro sistemático de las actividades de investigación desarrolladas por estos, permitiendo mantener una política de promoción y difusión de los investigadores de la UPEL.

35 La base de datos de los investigadores registrados en el RAIP-UPEL está disponible en la página del Vicerrectorado de Investigación y Postgrado, a través del enlace http://150.187.142.211/investigacion/investigadores.php. 
pública acerca de las actividades que realizan nuestros profesores en materia investigativa, lo cual puede ser verificado por cualquier integrante de la comunidad académica universitaria nacional o internacional.

Vinculación de las líneas de investigación con los programas de postgrado

Dentro de la perspectiva de la investigación en la UPEL, es importante destacar la organización de las líneas de investigación que se desarrollan en los Programas de Postgrado y su redireccionamiento hacia las Unidades de Investigación o entes académicos de la Universidad.

En ese sentido, en el mes de enero 2008, se realizó un primer encuentro denominado "Calidad y Pertinencia de la Investigación y el Postgrado en la Universidad Pedagógica a Experimental Libertador", en donde se generaron líneas de acción para impulsar los cambios en función de las exigencias nacionales e internacionales y se formularon acuerdos para la administración de la investigación que se realiza desde los postgrados.

\section{Evaluación del subprograma de Doctorado en Educación}

La Universidad Pedagógica Experimental Libertador, consciente de los cambios que se generan en los nuevos tiempos vinculados con transformaciones sociopolíticas y económicas, la elevada producción de información, el desarrollo científico tecnológico y el fenómeno de la globalización, entre otros, que demandan de las organizaciones plasticidad para adecuar sus misiones, objetivos y metas a nuevas condiciones; se planteó la revisión del Subprograma de Doctorado en Educación a partir de un proceso de autoanálisis reflexivo por parte de la comunidad académica que integra dicho Subprograma. Su finalidad estuvo dirigida a replantear el enfoque del mismo en términos de su orientación onto-epistemológica y su vinculación con la realidad sociocultural, económica y política local, regional y nacional, con una visión global, es así, como para el año 2006 se elaboró un diseño curricular en transición 2005/2010, el cual fue sometido a consideración del Consejo Universitario de la UPEL ${ }^{36}$.

Proceso de transformación curricular de los postgrados de la UPEL.

La propuesta de transformación curricular de los estudios de postgrado, surge de la necesidad sentida por la comunidad, pero también sugerida por los organismos nacionales e internacionales competentes en la materia.

\footnotetext{
${ }^{36}$ RUIZ, Carlos. (2007): Diseño Curricular del Doctorado en Educación. Universidad Pedagógica Experimental Libertador. Caracas: Vicerrectorado de Investigación y Postgrado.
} 
Es así que con dicha transformación se plantea estar en consonancia con las Políticas y Estrategias del Estado venezolano en materia de transformación universitaria ${ }^{37}$. Lo que se pretende es incorporar el currículo de postgrado a las nuevas tendencias y enfoques en los diseños que satisfagan la formación continua de sus egresados y de los profesionales de la docencia.

Asesoría permanente a las coordinaciones generales de investigación

Desde las Coordinaciones Nacionales del Vicerrectorado de Investigación y Postgrado, se generan de manera permanente, directrices para el acompañamiento de los profesores que se inician en el quehacer investigativo a través de las Coordinaciones Generales de Investigación; ello se ejecuta mediante asesorías para la elaboración de proyectos de investigación, la formulación adecuada de la distribución presupuestaria dentro de los proyectos de investigación financiados. Orientación acerca de los trámites para las solicitudes de financiamiento para actividades vinculadas con la investigación. De esta manera se pretende establecer un efecto sinérgico para la incorporación creciente de nuestros noveles profesores a la investigación.

\section{Programa de formación de tutores}

Se ha realizado un programa de formación de tutores con la finalidad de generar en los profesores investigadores un conjunto de competencias orientadas a la tutoría para el desarrollo de trabajos de grado; el mismo fue realizado bajo la modalidad a distancia.

\section{CONCLUSIÓN}

En el presente trabajo, a través de la visión histórica y estructural de la investigación en la UPEL, deseamos mostrar su evolución y estado actual.

Dada su responsabilidad con el país, en la UPEL existe una preocupación permanente por evaluar sus funciones en materia de Investigación. Es importante destacar que dado el crecimiento continuo de la población de docentes de la universidad, el interés progresivo por formarse como investigadores y la tendencia permanente de la universidad hacia la consolidación de su cultura investigativa, existe una revisión permanente de dichas actividades con el objeto de alcanzar una mayor eficiencia y eficacia.

37 Para ello el Vicerrectorado de Investigación y Postgrado ha emprendido un conjunto de acciones que tiene como primer producto un Informe de Avance con un diagnóstico de la situación a octubre de 2010 (Esteves, 2010). 
En base a los argumentos mostrados, podemos afirmar que la investigación en la UPEL aún transita por una fase de implementación; con una cultura investigativa en proceso de fortalecimiento y que tiende a instaurarse como parte del cotidiano quehacer de sus docentes investigadores, quienes a su vez, forman parte de la pléyade de investigadores que conforman el cuerpo de científicos sociales en nuestro país.

Para consolidar dicha cultura, es necesario continuar con el reto de mantener el ritmo de producción intelectual y de generación de nuevos conocimientos, para lo cual la asignación de recursos económicos por vía fiscal, es una demanda inaplazable.

Algunos de los lineamientos generales para el avance de la cultura investigativa, sugeridos son: continuar con el proceso de Integración de la Investigación con el Postgrado, seguir con el Programa de Formación de Tutores y de Formación de competencias investigativas, conformar Oficinas de Apoyo al investigador y continuar apoyando a los profesores y profesoras para la formulación de Proyectos de Investigación y asistencia a eventos de carácter nacional e internacional, recurriendo para ello a Políticas de Investigación sostenida con recursos fiscales y no propios de la universidad, entre otros.

Por último, para mejorar la investigación dentro de nuestra universidad; contamos con un capital humano conformado por noveles profesores investigadores a quienes se les debe apoyar a través de programas y actividades anteriormente referenciadas. Estos profesores constituyen la generación de relevo a quienes les corresponderá timonear esta gran nave, de allí que la perspectiva de la investigación está en sus manos, el camino está señalado ahora nos corresponde continuar con el andar.

\section{FUENTES}

Decreto $N^{\circ} 2176$, de fecha 28 de julio de 1983, Gaceta Oficial N .32 .777 de fecha 28 de julio de 1983.

LEY DE UNIVERSIDADES. (1970).

LEY ORGÁNICA DE EDUCACIÓN. (1980): Gaceta Oficial de la República de Venezuela, 2.635, julio 28.

LEY ORGÁNICA DE CIENCIA, TECNOLOGÍA E INNOVACIÓN (2005): Ministerio de Ciencia, Tecnología e Innovación.

UNIVERSIDAD PEDAGÓGICA EXPERIMENTAL LIBERTADOR. (1997): "Reglamento del Personal Académico". (Resolución N 97181 137, abril 26).

UNIVERSIDAD PEDAGÓGICA EXPERIMENTAL LIBERTADOR. (2003): "Reglamento General", Caracas. 
UNIVERSIDAD PEDAGÓGICA EXPERIMENTAL LIBERTADOR. (2004): "Manual de Trabajos de Grado de Especialización y Maestría y Tesis Doctorales", Caracas: FEDUPEL.

UNIVERSIDAD PEDAGÓGICA EXPERIMENTAL LIBERTADOR. (2004): "Catálogo de Líneas y Proyectos de Investigación", Caracas.

UNIVERSIDAD PEDAGÓGICA EXPERIMENTAL LIBERTADOR. (2004): Fichas Técnicas de las Unidades de Investigación, UPEL, Caracas.

UNIVERSIDAD PEDAGÓGICA EXPERIMENTAL LIBERTADOR. (2004): "Agenda Corporativa de Investigación y Postgrado", Caracas.

UNIVERSIDAD PEDAGÓGICA EXPERIMENTAL LIBERTADOR. (2007): "Plan de desarrollo 2007-2011". [Disponible en: www.upel.edu.ve. Consulta realizada el 03 Abril de 2008].

UNIVERSIDAD PEDAGÓGICA EXPERIMENTAL LIBERTADOR. (2008): "Política presupuestaria. Vicerrectorado de Investigación y Postgrado". No publicado.

UNIVERSIDAD PEDAGÓGICA EXPERIMENTAL LIBERTADOR. (2008): “Reglamento de Investigación”. (Resolución N²008.316.28.19, julio 23).

UNIVERSIDAD PEDAGÓGICA EXPERIMENTAL LIBERTADOR. (2009): "Política presupuestaria. Vicerrectorado de Investigación y Postgrado". No publicado.

VICERRECTORADO DE INVESTIGACIÓN Y POSTGRADO. (2008): "Informe del Congreso de Investigación en Educación. Retos y Acciones". No Publicado.

VICERRECTORADO DE INVESTIGACIÓN Y POSTGRADO. (2010): "La función de Investigación en la UPEL: pasado, presente y futuro". No publicado.

\section{REFERENCIAS}

BARRIOS, Maritza. (2006): "Vocaciones y Formación de Educadores", en: Doce propuestas educativas para Venezuela. Caracas, Fundación Polar/UCAB, Fondo de Publicaciones UCAB.

CORTÁZAR, José. (2008): Programa de Promoción al Investigador del Observatorio Nacional de Ciencia, Tecnología e Innovación. Ponencia presentada en la $2^{\circ}$ Reunión Ordinaria del Núcleo de los Consejo de Desarrollo Científico, Humanístico y Tecnológico (CDCHT). Universidad Nacional Experimental de los Llanos. Barinas, marzo, 27 y 28.

DE TEJADA, Miren. (2008): "Estructura organizativa de la investigación en la UPEL. Ponencia presentada en: evento Calidad y Pertinencia de la Investigación y el Postgrado de la Universidad Pedagógica Experimental Libertador'. Tucacas, trabajo no publicado.

DE TEJADA, Miren; SMITTER, Yajahira; MAZZARELLA, Clemen. (2009): "Vicerrectorado de Investigación y Postgrado". Informe de Gestión de la 
Coordinación Nacional del Programa de Investigación/Coordinación Nacional de FONDEIN. No Publicado.

ESTEVES, Moraima. (2010):“La transformación curricular en el Postgrado de la UPEL”, en: Informe de avances. Disponible: http://150.187.142.211/ documentos/archivos/Propuesta_de_estructura_para_transformacion_ curricular_octubre_10_.pdf [Consulta: 2011, Enero 05]

FONDO NACIONAL DE CIENCIA, TECNOLOGÍA E INNOVACIÓN. (2009): "Registro de Publicaciones Científicas y Tecnológicas Venezolanas FONACIT". Disponible: http://www.fonacit.gob.ve/verprintconvoca. asp?id $=68$ [Consulta: 2010, Enero 5]

GOVEA DE CARPIO, Duilia. (1989): Educación popular y formación docente, de la independencia al 23 de enero de 1958. Caracas, Academia Nacional de la Historia.

IZARRA, Douglas y ESCOBAR, Faviola. (2007): "Pertinencia de la investigación en los estudios de postgrado de la UPEL-IMPM", en: Investigación y Postgrado Vol. 22, N. 2.

MURO, Xiomara; NAVA, Minelia; GONZÁLEZ, María; GIMÉNEZ, Fanny; BECERRA, Osmery; DUBS, Renie; CALZADILLA, Omar; ARANA, Aracelis; OSORIO, Lesbia; QUINTERO, Carmen. (2003): "Hacia una definición de la calidad de la investigación en la Universidad Pedagógica Experimental Libertador", en: Investigación y Postgrado, Vol.18, N.1.

PEÑA, José. (2008): "Perspectivas de la Investigación en la UPEL. Ponencia presentada en: el foro Gestión de la Calidad en Educación; XII Jornada de Investigación Institucional de la UPEL-IPB. Caracas, Edo. Lara.

PEÑALVER, Luis. (2007): La Formación Docente en Venezuela. Caracas, Universidad Pedagógica Experimental Libertador, Vicerrectorado de Investigación y Postgrado.

ROJAS, Blanca y PÉREZ, Isabel. (2004): "Evaluación de la función de investigación en la UPEL-IPB”, en: Investigación y Postgrado, Vol.19, N. 2.

RUIZ, Carlos. (2007): Diseño Curricular del Doctorado en Educación. Caracas, Universidad Pedagógica Experimental Libertador. Vicerrectorado de Investigación y Postgrado.

SOSA, Elizabeth; SÁNCHEZ. Luis; DE TEJADA, Miren. (2010): "Propuesta de Integración entre la Investigación y el Postgrado, desde la visión del Instituto Pedagógico de Caracas". Subdirección de Investigación y Postgrado. No publicad.

PEÑA, José Alberto; DE TEJADA LAGONELL, Miren. (2011): "Evolución, avances y perspectivas de la investigación en una Universidad Pedagógica venezolana" en: Revista Historia de la Educación Latinoamericana Vol. 13 No. 17, Tunja, Universidad Pedagógica y Tecnológica de Colombia, RUDECOLOMBIA, SHELA- HISULA pp. 175-198 